\title{
Multicenter retrospective evaluation of the validity of the Thoracolumbar Injury Classification and Severity Score system in children
}

\author{
Jonathan N. Sellin, MD, ${ }^{1}$ William J. Steele III, MD, ${ }^{1}$ Lauren Simpson, MD, ${ }^{4}$ Wei X. Huff, MD, PhD, ${ }^{3}$ \\ Brandon C. Lane, MD, ${ }^{3}$ Joshua J. Chern, MD, PhD, ${ }^{2}$ Daniel H. Fulkerson, MD, ${ }^{3}$ \\ Christina M. Sayama, MD, MPH, ${ }^{4}$ and Andrew Jea, MD ${ }^{1}$ \\ 1Division of Pediatric Neurosurgery, Texas Children's Hospital, Department of Neurosurgery, Baylor College of Medicine, \\ Houston, Texas; 'Division of Pediatric Neurosurgery, Department of Neurosurgery, Emory University, Atlanta, Georgia; ${ }^{3}$ Division \\ of Pediatric Neurosurgery, Riley Children's Hospital, Indianapolis, Indiana; and ${ }^{4}$ Division of Pediatric Neurosurgery, Doernbecher \\ Children's Hospital, Portland, Oregon
}

\begin{abstract}
OBJECTIVE The Thoracolumbar Injury Classification and Severity Score (TLICS) system was developed to streamline injury assessment and guide surgical decision making. To the best of the authors' knowledge, external validation in the pediatric age group has not been undertaken prior to this report.
\end{abstract}

METHODS This study evaluated the use of the TLICS in a large retrospective series of children and adolescents treated at 4 pediatric medical centers (Texas Children's Hospital, Children's Healthcare of Atlanta, Riley Children's Hospital, and Doernbecher Children's Hospital). A total of 147 patients treated for traumatic thoracic or lumbar spine trauma between February 1, 2002, and September 1, 2015, were included in this study. Clinical and radiographic data were evaluated. Injuries were classified using American Spinal Injury Association (ASIA) status, Denis classification, and TLICS.

RESULTS A total of 102 patients (69\%) were treated conservatively, and 45 patients (31\%) were treated surgically. All patients but one in the conservative group were classified as ASIA E. In this group, 86/102 patients (84\%) had Denis type compression injuries. The TLICS in the conservative group ranged from 1 to 10 (mean 1.6). Overall, $93 \%$ of patients matched TLICS conservative treatment recommendations (score $\leq 3$ ). No patients crossed over to the surgical group in delayed fashion.

In the surgical group, $26 / 45$ (58\%) were ASIA E, whereas 19/45 (42\%) had neurological deficits (ASIA A, B, C, or D). One of $45(2 \%)$ patients was classified with Denis type compression injuries; $25 / 45$ (56\%) were classified with Denis type burst injuries; $14 / 45(31 \%)$ were classified with Denis type seat belt injuries; and $5 / 45(11 \%)$ were classified with Denis type fracture-dislocation injuries. The TLICS ranged from 2 to 10 (mean 6.4). Eighty-two percent of patients matched TLICS surgical treatment recommendations (score $\geq 5$ ). No patients crossed over to the conservative management group. Eight patients (8/147, 5\%) had a calculated TLICS of 4, which meant they were candidates for surgery or conservative therapy by TLICS criteria. Excluding these patients, the degree of agreement between TLICS and surgeon decision was deemed to be very good $(\kappa=0.878)$.

CONCLUSIONS The TLICS results and recommendations matched treatment in $96 \%$ of conservative group cases. In the surgical group, TLICS recommendations matched treatment in $93 \%$ of cases. The TLICS recommendations and surgeon decision making displayed very good concordance. The TLICS appears to be effective in the classification of thoracic and lumbar spine injuries and in guiding treatment in the pediatric age group.

http://thejns.org/doi/abs/10.3171/2016.1.PEDS15663

KEY WORDS pediatric spine; spine trauma; children; Thoracolumbar Injury Classification and Severity Score system; TLICS

ABBREVIATIONS ASIA = American Spinal Injury Association; MVA = motor vehicle accident; PLC = posterior ligamentous complex; SCIWORA = Spinal Cord Injury Without Radiographic Abnormality; TLICS = Thoracolumbar Injury Classification and Severity Score.

SUBMITTED November 10, 2015. ACCEPTED January 20, 2016.

INCLUDE WHEN CITING Published online April 8, 2016; DOI: 10.3171/2016.1.PEDS15663. 
A NUMBER of classification systems systematically describe thoracic and lumbar fractures. ${ }^{15}$ The Magerl-AO fracture classification is probably the most comprehensive and the most accurate. It has identified a wide variety of fractures and includes more than $50 \mathrm{sub}-$ types. Despite its importance, the Magerl-AO classification is rather onerous to apply in clinical practice, given its low inter- and intraobserver reliability. ${ }^{6,8,18}$

Most authors prefer to extrapolate from the Denis 3-column classification system, originally described for fractures at the thoracolumbar junction. ${ }^{2}$ The Denis spine stability classification divides the spine into 3 columns: anterior (i.e., ventral half of the vertebral body and intervertebral disc, which includes the anterior longitudinal ligament); middle (i.e., dorsal half of the vertebral body and intervertebral disc, which includes the posterior longitudinal ligament); and posterior (i.e., pedicles, lamina, facet joints, spinous processes, and interspinous and supraspinous ligaments). Denis described the 4 major types of injuries as compression, burst, seat belt, or fracturedislocation according to which a combination of columns suffered injury.

Several studies have demonstrated the Thoracolumbar Injury Classification and Severity Score (TLICS) system's good reliability and validity in terms of injury classification and treatment recommendations in the adult patient population. The TLICS system (Table 1) has also compared well with other thoracolumbar classification systems. ${ }^{11,12,17}$ However, most of these published studies were written by the original authors of the TLICS - with few attempts at external validation. ${ }^{3,4}$ Furthermore, authors have reported that the system is easily incorporated into clinical

TABLE 1. The TLICS system*

\begin{tabular}{lc}
\hline \multicolumn{1}{c}{ Feature } & Points \\
\hline Morphology type & 1 \\
\hline Compression & 2 \\
\hline Burst & 3 \\
\hline Translation/rotation & 4 \\
\hline Distraction & \\
\hline Neurological involvement & 0 \\
\hline Intact & 2 \\
\hline Nerve root & 2 \\
\hline Cord, conus medullaris (+1 point for incomplete injury) & 3 \\
\hline Cauda equina & 0 \\
\hline PLC & 2 \\
\hline Intact & 3 \\
\hline Injury suspected/indeterminate & \\
\hline Injured & $0-3$ \\
\hline Treatment recommendation & 4 \\
\hline Nonsurgical & $>4$ \\
\hline Surgeon's choice & \\
\hline Surgical &
\end{tabular}

* Modified from Koh YD, Kim DJ, Koh YW: Reliability and validity of Thoracolumbar Injury Classification and Severity Score (TLICS). Asian Spine J 4:109-117, 2010. CC BY-NC version 3.0 (http://creativecommons.org/licenses/ by-nc/3.0). practice and is a valuable tool in education. Nonetheless, it remains to be seen if TLICS is safe and effective in the pediatric patient population. Given the potential benefits of this new classification system, an assessment of the validity of TLICS is warranted. The purpose of our study was to evaluate use of TLICS in the pediatric population by retrospectively applying the TLICS to a group of children and adolescents who were treated for thoracic and lumbar injuries without pretreatment use of the TLICS system at 4 academic children's medical centers.

\section{Methods}

We retrospectively reviewed consecutive patients treated conservatively and surgically for thoracic and lumbar spine injuries between February 1, 2002, and September 1,2015 , at 4 academic children's medical centers. Patients were identified through prospective databases maintained independently at each center for all patients $\leq 18$ years of age in whom diagnoses of thoracic and/or lumbar injuries or spinal cord injuries had been made. Inclusion criteria also included complete clinical and radiological data (radiographs, CT scans, or MR images). Exclusion criteria included penetrating spine trauma, pathological fractures (cancer, osteoporosis, eosinophilic granuloma), spinous or transverse process fractures, patients with chronic or age-indeterminant thoracic and/or lumbar injuries, and patients in whom the TLICS was applied anytime during the hospital course as documented in their medical record.

The patients' neurological status was described using the American Spinal Injury Association (ASIA) classification system. Details of conservative treatment included use of external orthosis and duration of treatment. Surgical details included operative approach, number of operative levels, and intraoperative complications. Grading of spinal injuries was performed by the senior spine surgeon (J.J.C., D.H.F., C.M.S., and A.J.) from each institution, using available imaging modalities (radiographs, CT scans, and/or MR images). Although MRI is the gold standard for determining ligamentous injury, posterior ligamentous complex (PLC) injury may also be inferred on plain radiographs or CT scans by the degree of kyphotic angulation, splaying of adjacent spinous processes, diastasis of the facet joints, facet perch or subluxation, or vertebral body translation or rotation. ${ }^{18}$ Demographic data, including age and sex, were recorded. Baseline injury details were recorded, including cause of trauma, injury level, and neurological status. Injuries were described as thoracic (T1-9), thoracolumbar (T10-L2), or lumbar (L3-5). Using available radiographic studies, injuries were classified according to Denis and the TLICS at the most severely injured level. The Denis classification was chosen because it is popular and is commonly applied in clinical practice.

Surgical decision making was multifactorial, not standardized, and it was dependent on the individual senior surgeon. However, in general, surgeons shared a similar surgical philosophy. They used the Denis classification system to make treatment decisions, with injuries involving $\geq 2$ columns (burst, seat belt, and fracture-dislocation injuries) considered unstable. Compression fractures (injuries isolated to the anterior column) were considered 
stable and were managed conservatively. For burst fractures specifically, $>50 \%$ loss of body height, $>50 \%$ canal compromise, or $>30^{\circ}$ of kyphosis were relative indications for surgery. For seat belt fractures in particular, $>$ $17^{\circ}$ of kyphosis and predominant ligamentous disruption were relative indications for surgery. It must be emphasized that patients in this series were "TLICS-naïve." That is, the TLICS was not used to guide the surgeon's decision making in this patient population.

Outcomes of treatment were recorded, including postoperative complications (neurological deficit, crossover from conservative to surgical treatment, infection, fusion failure). Patients with failure in the initial conservative treatment group were maintained in the conservative group for analysis. The study was approved by the institutional review board of Baylor College of Medicine.

\section{Statistical Analysis}

Descriptive statistics, including mean, standard deviation, standard error, and confidence intervals were computed as necessary. Cohen's kappa coefficient was calculated to assess the correlation between the TLICS recommendation and the surgeon's decision making. Analysis was done using GraphPad Prism software.

\section{Results}

A total of 187 patients were initially identified and evaluated. Of these, 40 were excluded because of incomplete clinical or radiological data or because their injury was either minor (spinous or transverse process fracture) or not due to trauma (infectious or neoplastic). One hundred forty-seven patients met all inclusion and exclusion criteria. Forty-eight of 147 patients (33\%) were included from Texas Children's Hospital, 26/147 (18\%) from Children's Healthcare of Atlanta, 43/147 (29\%) from Riley Children's Hospital, and 30/147 (20\%) from Doernbecher Children's Hospital.

Forty-nine of 147 patients (33\%) had only CT scans available for analysis of their pattern of spine injury, 11/147 patients $(7 \%)$ had only MR images available, 69/147 patients (47\%) had both CT scans and MR images prepared for analysis, and 18/147 patients (12\%) had only radiographs readily available for study.

One hundred two patients (69\%) were treated conservatively, and 45 patients (31\%) were treated surgically. Ninety-eight patients $(67 \%)$ had a TLICS of $\leq 3 ; 8$ patients $(5 \%)$ had a TLICS of 4 ; and 41 patients $(28 \%)$ had a TLICS of $\geq 5$ (Table 2).

\section{Conservative Treatment Group}

The mean patient age was 12.7 years (range 1-18 years). Fifty-four patients (54/102, 53\%) were boys, and $48 / 102$ patients $(47 \%)$ were girls. The primary causes for injury, most commonly, were falls (54/102 patients, 53\%), followed by motor vehicle accident (MVA) (41/102 patients, 40\%) and blunt trauma/athletic competition (4/102 patients, $4 \%)$. Thirty-eight patients $(38 / 102,37 \%)$ had injuries in which the most severely injured level was in the thoracic spine; $42 / 102$ patients $(41 \%)$ had thoracolumbar injuries; and 22/102 patients (22\%) had lumbar injuries
TABLE 2. Comparison of patient treatment (conservative versus surgical) and TLICS score

\begin{tabular}{crcc}
\hline & \multicolumn{3}{c}{ TLICS Score } \\
\cline { 2 - 4 } Treatment & $1-3$ & 4 & $\geq 5$ \\
\hline Conservative group & 95 & 3 & 4 \\
\hline Surgical group & 3 & 5 & 37 \\
\hline
\end{tabular}

(Table 3). All but 1 patient were ASIA E on presentation (99\%). Thirty-six patients $(36 / 102,35 \%)$ were treated with no bracing, whereas $64 / 102$ patients $(63 \%)$ were treated with an external orthosis for as long as 12 weeks. Bracing status was unknown for 2 patients.

Follow-up was available for 79/102 patients (77\%). The median follow-up for patients in this treatment group was 4 months (range 0.6-95 months). No patients demonstrated neurological deterioration. One case of delayed scoliosis, which did not require surgery, was noted as a complication in the conservative group $(1 / 102,1 \%)$. No patients for whom follow-up was available required surgery in delayed fashion.

Fracture type according to the Denis classification included $86 / 102$ patients $(84 \%)$ with compression fracture; $12 / 102$ patients (12\%) with burst fractures; and 4/102 patients (4\%) with seat belt injuries. No patients had Denis type fracture-dislocation injuries.

The TLICS ranged from 1 to 10 (mean 1.6) in this conservative group (Table 4). In 98 of 102 patients (96\%), treatment matched the TLICS recommendations for conservative treatment (score $\leq 3$ or $=4$ ).

\section{Surgical Treatment Group}

The mean patient age was 13.5 years (range $2-18$ years). Nineteen patients $(19 / 45,42 \%)$ were boys, and $26 / 45$ pa-

TABLE 3. Distribution of the 147 patients included in the study according to treatment

\begin{tabular}{|c|c|c|}
\hline \multirow[b]{2}{*}{ Characteristic } & \multicolumn{2}{|c|}{ No. $(\%)$} \\
\hline & Conservative Group & Surgical Group \\
\hline \multicolumn{3}{|l|}{ Injury location } \\
\hline Thoracic (T1-9) & $38(37)$ & $8(18)$ \\
\hline Thoracolumbar (T10-L2) & $42(41)$ & $22(49)$ \\
\hline Lumbar (L3-5) & $22(22)$ & $15(33)$ \\
\hline \multicolumn{3}{|l|}{ Mechanism } \\
\hline MVA & $41(40)$ & $31(69)$ \\
\hline Fall & $54(53)$ & $10(22)$ \\
\hline Blunt trauma & $4(4)$ & $3(7)$ \\
\hline \multicolumn{3}{|l|}{ ASIA score } \\
\hline$E$ & $101(99)$ & $26(58)$ \\
\hline$A, B, C$, or $D$ & $1(1)$ & $19(42)$ \\
\hline \multicolumn{3}{|l|}{ Denis fracture type } \\
\hline Compression & $86(84)$ & $1(2)$ \\
\hline Burst & $12(12)$ & $25(56)$ \\
\hline Seat belt/Chance & $4(4)$ & $14(31)$ \\
\hline Fracture/dislocation & $0(0)$ & $5(11)$ \\
\hline Total & 102 & 45 \\
\hline
\end{tabular}


tients $(58 \%)$ were girls. The primary etiologies for injury were MVA (31/45 patients, $69 \%)$, followed by falls (10/45 patients, 22\%) and blunt trauma/athletic competition (3/45 patients, $7 \%$ ). The most severe injury was found in the thoracic spine for $8 / 45$ patients (18\%); 22/45 patients (49\%) had thoracolumbar injuries; and 15/45 patients (33\%) had lumbar injuries (Table 3$)$. Twenty-six patients $(26 / 45,58 \%)$ in the surgical group were ASIA E, whereas $19 / 45$ patients (42\%) had neurological deficits (ASIA A, B, C, or D).

Follow-up was available for all 45 surgical patients. The median follow-up for patients in this treatment group was 9 months (range 1-161 months). No patients demonstrated neurological deterioration. There were 4 intraoperative or postoperative complications in the surgical cohort $(4 / 45,9 \%)$. These complications included 2 patients with CSF leaks, 1 patient with a new postoperative neurological deficit, and 1 patient with persistence of preoperative neurogenic bladder and lower-extremity dysesthesias. The patient with the new neurological deficit after surgery was a 16-year-old girl who presented with an L-3 burst fracture and right distal tibia fracture. The patient was brought to the operating room for posterior lumbar fusion and open reduction and internal fixation of the tibia. The examination of the right distal lower extremities was limited before surgery. After surgery the patient was in a cast, and complained of foot numbness that resolved within a few weeks. Foot numbness was most likely related to the cast or to positioning of the patient during surgery. However, because of the uncertainty about the cause of the foot numbness, we deemed this a complication of surgery. No patients required reoperation following surgical management.

One of 45 patients (2\%) was classified with Denis type compression injuries; $25 / 45$ patients $(56 \%)$ were classified with Denis type burst injuries; $14 / 45$ patients (31\%) were classified with Denis type seat belt injuries; and 5/45 patients $(11 \%)$ were classified with Denis type fracturedislocation injuries.

The TLICS ranged from 2 to 10 (mean 6.4). Forty-two of 45 (93\%) patients matched TLICS surgical treatment recommendations (score $\geq 5$ or $=4$ ). No patients crossed over to the conservative management group (Table 4).

\section{The TLICS Conservative Group (TLICS $\leq 3$ )}

Ninety-eight patients $(98 / 147,67 \%)$ had a TLICS of $\leq 3$. The mean patient age was 12.6 years (range 1.0-18 years). Fifty patients $(50 / 98,51 \%)$ were boys, and $48 / 98$ patients $(49 \%)$ were girls. The primary etiologies for injury, most commonly, were falls (53/98 patients, 54\%), followed by MVA (38/98 patients, 39\%) and blunt trauma/athletic competition (4/98 patients, $4 \%)$. Thirty-six patients (36/98, $37 \%$ ) had injuries in which the most severely injured level was in the thoracic spine; 41/98 (42\%) had thoracolumbar injuries; and 21/98 (21\%) had lumbar injuries. All but 1 patient were ASIA E on presentation (99\%). Fracture type according to the Denis classification included $86 / 98$ patients (88\%) with compression fracture and 12/98 patients (12\%) with burst fractures. No patients had Denis type seat belt or fracture-dislocation injuries (Table 5).

Ninety-five of these patients (97\%) were managed conservatively, with 3 of 98 cases (3\%) managed surgically.
TABLE 4. Distribution of the 147 patients treated according to TLICS score

\begin{tabular}{crr}
\hline TLICS Score & No. of Patients & $\%$ \\
\hline Conservative group & & \\
\hline $1-3$ & 95 & 93 \\
\hline 4 & 3 & 3 \\
\hline 25 & 4 & 4 \\
\hline Total & 102 & 100 \\
\hline Surgical group & & 7 \\
\hline $1-3$ & 3 & 11 \\
\hline 4 & 5 & 82 \\
\hline$\geq 5$ & 37 & 100 \\
\hline Total & 45 &
\end{tabular}

Of the 95 conservatively managed patients, $36(38 \%)$ were treated with no bracing, whereas 58 patients (61\%) were treated with an external orthosis for as long as 12 weeks. Bracing status was unknown for 1 of the 95 patients. Thus, in $95 \%$ of cases in which the TLICS was $\leq 3$, treatment matched the TLICS recommendations.

\section{The TLICS Intermediate Group (TLICS 4)}

Eight patients $(8 / 147,5 \%)$ had a calculated TLICS of 4, meaning they were candidates for surgery or conservative therapy by TLICS criteria. The mean patient age was 13.1 years (range 3-16 years). Six patients (75\%) were boys. The primary causes for injury were MVA (5/8 patients, $62.5 \%)$, followed by falls (2/8 patients, $25 \%)$ and blunt trauma/athletic competition (1/8 patients, $12.5 \%)$. One patient $(12.5 \%)$ had an injury most severe at the thoracic level; 5/8 patients (62.5\%) had thoracolumbar injuries; and

TABLE 5. Distribution of the 147 patients included in the study according to TLICS score

\begin{tabular}{|c|c|c|c|}
\hline \multirow[b]{2}{*}{ Characteristic } & \multicolumn{3}{|c|}{ TLICS Score, No. (\%) } \\
\hline & $\leq 3$ & 4 & $\geq 5$ \\
\hline \multicolumn{4}{|l|}{ Injury location } \\
\hline Thoracic (T1-9) & $36(37)$ & $1(12.5)$ & $9(22)$ \\
\hline Thoracolumbar (T10-L2) & $41(42)$ & $5(62.5)$ & $21(51)$ \\
\hline Lumbar (L3-5) & $21(21)$ & $2(25)$ & $11(27)$ \\
\hline \multicolumn{4}{|l|}{ Mechanism } \\
\hline MVA & $38(39)$ & $5(62.5)$ & $29(71)$ \\
\hline Fall & $53(54)$ & $2(25)$ & $9(22)$ \\
\hline Blunt trauma & $4(4)$ & $1(12.5)$ & $2(5)$ \\
\hline \multicolumn{4}{|l|}{ ASIA score } \\
\hline$E$ & $97(99)$ & $7(87.5)$ & $22(54)$ \\
\hline$A, B, C$, or $D$ & $1(1)$ & $1(12.5)$ & $19(46)$ \\
\hline \multicolumn{4}{|l|}{ Denis fracture type } \\
\hline Compression & $86(88)$ & $0(0)$ & $1(2)$ \\
\hline Burst & $12(12)$ & $7(87.5)$ & $18(44)$ \\
\hline Seat belt/Chance & $0(0)$ & $1(12.5)$ & $17(41)$ \\
\hline Fracture/dislocation & $0(0)$ & $0(0)$ & $5(12)$ \\
\hline Total & 98 & 8 & 41 \\
\hline
\end{tabular}


2/8 patients $(25 \%)$ had lumbar injuries. All patients with documented exams (7/8) were ASIA E. Fracture classification according to the Denis classification included $7 / 8$ patients $(87.5 \%)$ with burst fracture; $1 / 8$ patients $(12.5 \%)$ with seat belt injuries; and no patients with compression or fracture-dislocation injuries (Table 5).

Inherently, given the treatment recommendation of a TLICS of 4, treatment matched TLICS recommendations $100 \%$ of the time. Three patients $(3 / 8,37.5 \%)$ were treated conservatively in this intermediate group, and $5 / 8$ patients $(62.5 \%)$ were treated surgically. Of the 3 conservatively treated patients, 1 (33\%) was treated with no bracing, whereas $2(66 \%)$ were treated with an external orthosis for up to 12 weeks.

\section{The TLICS Surgical Group (TLICS $\geq 5$ )}

Forty-one of 147 patients (28\%) had a TLICS of $\geq 5$. The mean patient age was 13.6 years (range 1-18 years). Seventeen of these 41 patients (41\%) were boys, and 24 $(59 \%)$ were girls. The primary causes for injury were MVA (29/41 patients, $71 \%$ ), followed by falls (9/41 patients, $22 \%$ ) and blunt trauma/athletic competition (2/41 patients, 5\%). The most severe injury was found in the thoracic spine for $9 / 41$ patients (22\%); $21 / 41$ patients $(51 \%)$ had thoracolumbar injuries; and 11/41 patients (27\%) had lumbar injuries. Twenty-two of 41 patients (54\%) in the surgical group were ASIA E, whereas 19 (46\%) had neurological deficits (ASIA A, B, C, or D). One of 41 patients (2\%) was classified with Denis type compression injuries; $18 / 41$ patients (44\%) were classified with Denis type burst injuries; $17 / 41$ patients (41\%) were classified with Denis type seat belt injuries; and 5/41 patients (12\%) were classified with Denis type fracture-dislocation injuries (Table 5).

Of these 41 patients, 4 (10\%) were managed conservatively, whereas $37 / 41$ patients $(90 \%)$ were managed surgically. Thus, in $90 \%$ of instances where the TLICS was $\geq$ 5 , surgical treatment matched TLICS recommendations.

\section{Concordance Between TLICS and Treatment}

Excluding the patients with a TLICS of 4 (in whom either conservative or surgical treatment is acceptable), the degree of agreement between the TLICS and the surgeon's decision was calculated to be very good $(\kappa=0.878)$.

\section{Discussion}

The mechanism of spinal injury in children varies with age. The major causes of injury in young children ages 0-9 years are falls and automobile-versus-pedestrian accidents (> 75\%).,15,19 An MVA (40\%) is the leading cause of lumbar fractures in children ages 10-14 years; in this age group, falls and automobile-versus-pedestrian accidents are less prevalent. ${ }^{15}$ Motor vehicle and motorcycle accidents are the predominant causes of spine injuries (> $70 \%$ ) in adolescents ages 15-17 years; in this age group, there is also an increase in sports-related spine trauma. ${ }^{15}$ Our study findings generally matched these reported demographics of pediatric spine injury, with $49 \%(72 / 147)$ of injuries in our cohort caused by MVA and 44\% (64/147) of injuries caused by falls.

The pediatric spinal column differs from the adult spine in several ways. The proportionally larger heads of infants and young children compared with their bodies, as well as their underdeveloped neck musculature, can make them more prone to flexion and extension injuries. ${ }^{13,15}$ Infants and small children also have inherent ligamentous laxity, elasticity, and incomplete ossification. ${ }^{9,12,15}$ Small facet joints that are more horizontally oriented result in greater mobility and less stability., ${ }^{9,10,15,16}$ Younger children $(0-8$ years) tend to have fewer fractures and greater incidence of Spinal Cord Injury Without Radiographic Abnormality (SCIWORA) due to these biomechanical differences. Hyperextension, together with the hypermobility of the pediatric spine, can cause momentary dislocation followed by spontaneous reduction. This may result in a damaged spinal cord but a radiographically normal-appearing vertebral column.7,915 A SCIWORA has been reported to occur in as high as $20 \%$ of children, but falls dramatically to $<1 \%$ in adults. An adult-like vertebral column (9-16 years), which has sturdier osseoligamentous formation, provides better spinal cord protection; thus, children and adolescents experience less severe spinal cord injury than their younger counterparts. Moreover, the adult spine, when subjected to high stress, is more prone to suffering breakage of bones and frank rupture of ligaments compared with the pediatric spine, in which deformation and return to normal alignment is more common.

Different classification schemes exist for thoracic and lumbar spine fractures; ${ }^{15}$ none have been validated in published works in children and adolescents. The TLICS has commonly been applied in the adult trauma population to help determine nonoperative versus operative management of thoracolumbar fractures. It has subsequently been studied and applied for thoracic fractures and/or lumbar fractures alone. A score is calculated based on injury mechanism, neurological status, and integrity of the PLC: a score of $\leq 3$ suggests nonoperative treatment; a score of 4 suggests either nonoperative or operative treatment; and a score of $\geq 5$ suggests operative treatment. ${ }^{5}$ The TLICS system has previously been externally validated in the adult population, most notably by Joaquim et al. ${ }^{3}$ In their retrospective series of 458 patients, TLICS recommendations matched treatment in $307 / 310$ patients (99\%) in the conservative, nonoperative cohort, whereas only 69/148 patients $(46.6 \%)$ showed similar agreement in the surgical cohort. It should be noted that all patients in the surgical group whose treatment was discordant with their score were those with a burst fracture that would have been classified as stable under TLICS guidelines, but who received operative therapy in practice.

The TLICS has recently been studied in a preliminary fashion in the pediatric population. In 2013, Garber et al. reported excellent validity of this scoring system in their pediatric cohort in an oral presentation (Garber ST, Rozelle C, Walker M: The Thoracolumbar Injury Classification and Severity Score in children: a validity study). The aforementioned structural differences between the pediatric spinal column and adult spine, combined with the paucity of published literature on the reliability and validity of the TLICS in the pediatric age group, underline the significance of a multicentered external validation of the spine injury classification system focused on children. 
Our study demonstrated that the TLICS accurately correlates with a surgeon's decision to offer conservative or surgical management. A total of 102 of 147 patients (69\%) were treated conservatively. Treatment was successful in all patients who were treated conservatively, either with or without bracing. It is important to note that, for those patients with follow-up (77\%), no patients in the conservative group crossed over to the late surgical group in long-term follow-up. On the other hand, a total of 45 patients (31\%) were treated surgically on initial presentation. Follow-up was available for all patients in the surgical cohort. No patients required reoperation. Complications rates were low in both cohorts (1\% vs $9 \%$ ), but were more prevalent in the surgical group. There were no deaths in either cohort. No patients in the conservative group experienced new neurological deficits or decline, as opposed to 1 patient in the surgical group $(1 / 45,2 \%)$.

Not surprisingly, the surgical group was characterized by a higher percentage of MVAs, neurological deficits (ASIA A, B, C, or D), and Denis type seat belt or fracturedislocation injuries $(69 \%, 42 \%$, and $42 \%$, respectively) than in the conservative group $(40 \%, 1 \%$, and $4 \%$, respectively). Also, as expected, the mean TLICS was higher in the surgical group than in the conservative group (6.4 vs 1.6). Excluding the patients with an intermediate TLICS of 4 (in whom either conservative or surgical treatment is acceptable), the degree of agreement between the TLICS and the surgeon's decision was calculated to be very good $(\kappa=0.878)$.

\section{Limitations of the Study}

Despite the involvement of multiple children's medical centers and a large number of patients, our study is limited by its retrospective nature. Retrospective application of the TLICS can demonstrate validity; however, it does not demonstrate safety and efficacy. We had limited objective measurements of long-term patient outcomes; healthrelated quality of life measures in particular.

Furthermore, we cannot determine how use of the TLICS impacts patient care because it was applied retrospectively rather than being used as part of the concurrent decision-making process. Importantly, because we were not blinded to treatment, potential bias in assessing the score for surgical and conservative treatment cannot be accounted for and eliminated.

Additionally, surgeons' decision making probably varied over time and between institutions. One might argue, however, that evolving decision making in the senior surgeons, as well as the diversity in decision making between the senior surgeons, accurately mirrors real practice. The fact that the TLICS system was remarkably faithful to actual treatment recommendations, from several different surgeons, over an extended period of time, argues in favor of its broad applicability.

Finally, clinical follow-up in our series was limited, with median follow-up times for conservative and surgical groups of only 4 and 9 months, respectively. Our report of clinical complications may not include significant delayed complications that were not captured by our limited follow-up periods.

\section{Conclusions}

Our study was the first application of the TLICS to a large number of consecutive children and adolescents at several children's medical centers. Because it demonstrated high concordance between surgeon decision making and TLICS in regard to nonoperative and operative treatment, the TLICS may indeed be a valuable, valid, and reliable tool in the rapid evaluation of pediatric thoracic and lumbar spine injuries. Prospective application of the TLICS in an organized prospective clinical trial may better elucidate the incremental differences between surgical and conservative management in the pediatric age group.

\section{References}

1. Akbarnia BA: Pediatric spine fractures. Orthop Clin North Am 30:521-536, x, 1999

2. Denis F: The three column spine and its significance in the classification of acute thoracolumbar spinal injuries. Spine (Phila Pa 1976) 8:817-831, 1983

3. Joaquim AF, Daubs MD, Lawrence BD, Brodke DS, Cendes $\mathrm{F}$, Tedeschi H, et al: Retrospective evaluation of the validity of the Thoracolumbar Injury Classification System in 458 consecutively treated patients. Spine J 13:1760-1765, 2013

4. Joaquim AF, Fernandes YB, Cavalcante RA, Fragoso RM, Honorato DC, Patel AA: Evaluation of the thoracolumbar injury classification system in thoracic and lumbar spinal trauma. Spine (Phila Pa 1976) 36:33-36, 2011

5. Lee JY, Vaccaro AR, Lim MR, Oner FC, Hulbert RJ, Hedlund $\mathrm{R}$, et al: Thoracolumbar injury classification and severity score: a new paradigm for the treatment of thoracolumbar spine trauma. J Orthop Sci 10:671-675, 2005

6. Magerl F, Aebi M, Gertzbein SD, Harms J, Nazarian S: A comprehensive classification of thoracic and lumbar injuries. Eur Spine J 3:184-201, 1994

7. Marar BC: Hyperextension injuries of the cervical spine. The pathogenesis of damage to the spinal cord. J Bone Joint Surg Am 56:1655-1662, 1974

8. Oner FC, Ramos LM, Simmermacher RK, Kingma PT, Diekerhof CH, Dhert WJ, et al: Classification of thoracic and lumbar spine fractures: problems of reproducibility. A study of 53 patients using CT and MRI. Eur Spine J 11:235-245, 2002

9. Pang D, Pollack IF: Spinal cord injury without radiographic abnormality in children - the SCIWORA syndrome. J Trauma 29:654-664, 1989

10. Pang D, Wilberger JE Jr: Spinal cord injury without radiographic abnormalities in children. J Neurosurg 57:114-129, 1982

11. Patel AA, Vaccaro AR, Albert TJ, Hilibrand AS, Harrop JS, Anderson DG, et al: The adoption of a new classification system: time-dependent variation in interobserver reliability of the thoracolumbar injury severity score classification system. Spine (Phila Pa 1976) 32:E105-E110, 2007

12. Raja Rampersaud Y, Fisher C, Wilsey J, Arnold P, Anand N, Bono CM, et al: Agreement between orthopedic surgeons and neurosurgeons regarding a new algorithm for the treatment of thoracolumbar injuries: a multicenter reliability study. J Spinal Disord Tech 19:477-482, 2006

13. Reddy SP, Junewick JJ, Backstrom JW: Distribution of spinal fractures in children: does age, mechanism of injury, or gender play a significant role? Pediatr Radiol 33:776-781, 2003

14. Roche C, Carty H: Spinal trauma in children. Pediatr Radiol 31:677-700, 2001

15. Sayama C, Chen T, Trost G, Jea A: A review of pediatric lumbar spine trauma. Neurosurg Focus 37(1):E6, 2014

16. Swischuk LE, Swischuk PN, John SD: Wedging of C-3 in 
infants and children: usually a normal finding and not a fracture. Radiology 188:523-526, 1993

17. Vaccaro AR, Baron EM, Sanfilippo J, Jacoby S, Steuve J, Grossman E, et al: Reliability of a novel classification system for thoracolumbar injuries: the Thoracolumbar Injury Severity Score. Spine (Phila Pa 1976) 31 (11 Suppl):S62-S69, S104, 2006

18. Vaccaro AR, Lehman RA Jr, Hurlbert RJ, Anderson PA, Harris M, Hedlund R, et al: A new classification of thoracolumbar injuries: the importance of injury morphology, the integrity of the posterior ligamentous complex, and neurologic status. Spine (Phila Pa 1976) 30:2325-2333, 2005

19. Vialle LR, Vialle E: Pediatric spine injuries. Injury 36 (Suppl 2):B104-B112, 2005

\section{Disclosures}

The authors report no conflict of interest concerning the materials or methods used in this study or the findings specified in this paper.

\section{Author Contributions}

Acquisition of data: all authors. Analysis and interpretation of data: Jea, Sellin. Drafting the article: Jea, Sellin. Critically revising the article: Jea, Fulkerson, Sayama. Approved the final version of the manuscript on behalf of all authors: Jea. Study supervision: Jea.

\section{Supplemental Information}

Previous Presentations

Portions of this study were presented as an oral report at the meeting of the AANS/CNS Section on Pediatric Neurological Surgery, held in Toronto, Canada, in 2013.

\section{Correspondence}

Andrew Jea, Division of Pediatric Neurosurgery, Texas Children's Hospital, 6621 Fannin St., CCC 1230.01, 12th Fl., Houston, TX 77030. email: ahjea@texaschildrens.org. 